Rechtsmedizin $2022 \cdot 32: 195-200$

https://doi.org/10.1007/s00194-021-00512-6

Angenommen: 29. Mai 2021

Online publiziert: 6 . Juli 2021

() Der/die Autor(en) 2021

\section{Eine retrospektive Untersuchung zu differenzialdiagnostischen Überlegungen in vermeintlichen Kinderschutzfällen}

\author{
Kindeswohlgefährdung, klarer Fall - oder?
}

\author{
Stefanie Röding ${ }^{1}$. Aline Dittmann-Wolf ${ }^{2}$. Denise Lackner ${ }^{3}$. Christian Blume ${ }^{4}$. \\ Anette Hasse ${ }^{1,2} \cdot$ Elisabeth Mützel $^{3} \cdot$ Gerhard K. Wolf' \\ 'Kinderklinik Traunstein, Kliniken Südostbayern, akademisches Lehrkrankenhaus der LMU München, \\ Traunstein, Deutschland \\ ${ }^{2}$ Sozialpädiatrisches Zentrum, Kliniken Südostbayern, akademisches Lehrkrankenhaus der LMU München, \\ Traunstein, Deutschland \\ ${ }^{3}$ Institut für Rechtsmedizin, Ludwig-Maximilians-Universität München, München, Deutschland \\ ${ }^{4}$ Kinderchirurgie und Kinderurologie Südostbayern, Traunstein, Deutschland
}

\title{
Zusammenfassung
}

Hintergrund: Die Diagnosestellung einer Kindesmisshandlung kann mit Irrtümern in beide Richtungen verbunden sein; einerseits können Misshandlungen unentdeckt bleiben oder aber Misshandlungen fälschlicherweise als Ursache für Verletzungen angenommen werden. Derartige diagnostische Irrtümer können dazu führen, dass Kinder dem misshandelnden Umfeld weiterhin ausgesetzt werden oder aber Kinder zu Unrecht in Obhut genommen und von ihrer Familie getrennt werden.

Methoden: Kinderschutzfälle aus den letzten 10 Jahren wurden retrospektiv anhand der elektronischen Krankenakte auf falsch-positive Fälle untersucht. Ein positives Ethikvotum der LMU München zur Publikation liegt vor.

Ergebnisse: Die als falsch-positiv identifizierten Fälle hatten als Ursache für vermeintliche nichtakzidentelle Verletzungsmuster eine genetisch gesicherte Osteogenesis imperfecta Typ 6, einen Lichen sclerosus und eine Hämophilie B. In allen Fällen wurde das Jugendamt miteinbezogen, in allen Fällen kam es zu einer Belastung für Eltern und Kinder, in einem Fall kam es zu einer, im Nachhinein ungerechtfertigten, temporären Inobhutnahme.

Diskussion: Eine interdisziplinär und multiprofessionell besetzte Kinderschutzgruppe an der Kinderklinik kann notwendige Differenzialdiagnosen ins Gespräch bringen und die Koordination mit dem Jugendamt und der Polizei erleichtern. Die Inobhutnahme durch das Jugendamt ist einerseits eine wichtige Maßnahme, um Kinder und Jugendliche vor weiteren Gefährdungen zu schützen, andererseits sollten ungerechtfertigte Inobhutnahmen vermieden werden, weil die daraus resultierende Trennung von Eltern und Kind zu erheblichen Belastungen führen kann. Die Konsultation der Rechtsmedizin ist hilfreich, um die Diagnose entsprechend einzugrenzen oder aber um alternative, medizinische Diagnosen anzubieten.

\section{Schlüsselwörter}

Kindesmisshandlung · Kinderschutzgruppe · Osteogenesis imperfecta · Lichen sclerosus . Hämophilie 


\section{Einleitung}

Kinderschutz kann häufig nur interdisziplinär geregelt werden, indem multiple Fachdisziplinen miteinander kooperieren. An vielen deutschen Kinderkliniken bestehen inzwischen Kinderschutzgruppen, welche interdisziplinär besetzt und in der Regel mit dem Jugendamt, der Polizei, dem Familiengericht und der Rechtsmedizin vernetzt sind. Am beschriebenen Standort ist die Kinderschutzgruppe nach den Empfehlungen der Deutschen Gesellschaft für Kinderschutz in der Medizin multidisziplinär zusammengesetzt (Kinderheilkunde, Kinderchirurgie, Kinderneurologie, Radiologie, Psychotherapie, Sozialdienst und Pflege) und kooperiert mit dem Institut für Rechtsmedizin der LMU. Das Ziel des Zusammenwirkens dieser verschiedenen Fachdisziplinen ist es, Kindeswohlgefährdungen zu erkennen und ggf. adäquate Schutzmaßnahmen für das Kind einzuleiten. Die Bewertung, ob eine Kindeswohlgefährdung vorliegt oder nicht, ist komplex, und die medizinische Diagnosestellung erfordert eine hohe fachliche Expertise. Dass kleine Kinder den Verletzungshergang oftmals nur begrenzt beschreiben können, teilweise Sprachbarrieren existieren und Eltern oder andere betreuende Personen eine Misshandlung in der Regel nicht offen ansprechen und einräumen, erschwert die Diagnosestellung zusätzlich.

Einerseits müssen Kinder, deren Wohl gefährdet ist, umgehend geschützt werden, andererseits bedeuten ungerechtfertigte Verdächtigungen und falsch-positive Einschätzungen von Anzeichen für eine Kindeswohlgefährdung einen schweren Schaden für Kinder und Eltern, den es ebenso zu vermeiden gilt. Vor diesem Hintergrund müssen neben medizinischen Diagnosen, die den Verdacht einer Misshandlung oder eines sexuellen Missbrauchs bestätigen oder auch widerlegen, auch stets alternative Erklärungen für scheinbare Misshandlungen in Erwägung gezogen werden. Steht die Verdachtsdiagnose "Kindesmisshandlung" einmal im Raum, kommt es häufig zu einem gravierenden Vertrauensverlust zwischen Ärztinnen/Ärzten und Eltern.

Fatalerweise kommt es immer wieder zu folgenschweren Fehleinschätzungen: Einerseits bleiben Kindesmisshandlungen manchmal unentdeckt, weil die Diagnose aus verschiedenen Gründen nicht richtig gestellt werden konnte, das Kind kommt nach der klinischen Vorstellung in das familiäre Setting zurück, und die Misshandlungen setzen sich fort. Andererseits liegt in manchen Fällen gar keine Misshandlung oder ein Missbrauch eines Kindes vor, und Erkrankungen, die zu den entsprechenden "Verletzungsmustern" führten, werden nicht erkannt. Diese Fälle werden aufgrund einer Fehlinterpretation der Befunde möglicherweise als Kindeswohlgefährdung dem Jugendamt mitgeteilt, was dazu führen kann, dass die Kinder von den Fachkräften des Jugendamtes (dann ungerechtfertigt) in Obhut genommen werden und sich eine notwendige Therapie der Grunderkrankung verzögert. Auch in diesem Fall wird das Wohl des Kindes gefährdet, jedoch ergibt sich die (sekundäre) Kindeswohlgefährdung [5] aus dem Handeln der professionellen Akteure und Akteurinnen.

Einige dieser medizinischen Befunde und differenzialdiagnostischen "Erkrankungen“, welche als Erklärung für Hämatome, Blutungen, Frakturen etc. dienen können, sollen vorgestellt und kritisch gewürdigt werden.

\section{Methoden}

Die Kinderschutzfälle der letzten 10 Jahre wurden revidiert. Kinderschutzfälle $(n=33)$, welche im Rahmen der klinischen Routine zur Vorstellung kamen, wurden identifiziert. In etwa der Hälfte der Fälle lag eine eindeutige Kindesmisshandlung vor, in 6 dieser Fälle kam es zur Inobhutnahme. In den anderen Fällen wurde zwar keine Kindesmisshandlung, aber zumindest eine Vernachlässigung festgestellt. Ein Kind war bereits vor der klinischen Vorstellung vom Jugendamt in Obhut genommen. Bei 2 Verdachtsfällen wurde die eindeutige Diagnose erst nach erneuter Vorstellung gestellt. Die Bayerische Kinderschutzambulanz der Rechtsmedizin der LMU München wurde bei Unklarheiten sekundär entweder telefonisch oder über ein Online-Portal („Remed-online“ [1]) konsultiert. Die Anamnesen und Darstellungen (z. B. Alter) wurden an bestimmten Stellen verfremdet, um eine nachträgliche Identifikation auszuschließen. Es besteht ein positives Ethikvotum der Ethikkommission der LMU München zur Publikation dieser Fälle.

\section{Ergebnisse}

Fall 1

Ein 15 Monate altes Kind wurde von seinen Eltern mit seit 2 Tagen bestehenden Schmerzen und Schwellung am linken Oberschenkel in der Notaufnahme vorgestellt. Radiologisch zeigte sich eine linksseitige Femurfraktur, die kinderchirurgisch mit Prévot-Nägeln versorgt wurde.

Die Familie war vor wenigen Jahren nach Deutschland geflüchtet. Auch im ausführlichen Gespräch mit dem Dolmetscher können die Eltern keine adäquate Erklärung für die Fraktur liefern. Das Kind laufe seit ca. 2 Monaten frei und sei vor 2 Tagen aus dem Stand auf den Po gefallen, als inn seine Mutter gebeten habe, aus dem Weg zu gehen. Seither laufe es nicht mehr und sei insgesamt sehr weinerlich.

Bei der Durchsicht der Krankenakten (pädiatrisch, kinder- und unfallchirurgisch) fiel auf, dass das Kind bereits 2 Monate zuvor aufgrund einer Oberarmfraktur links in konservativer kinderchirurgischer Behandlung war. Ursache sei der Sturz von einem Hocker nach Schubsen durch ein Geschwisterkind gewesen. Vor 3 Wochen war eine unfallchirurgische Vorstellung aufgrund von Schmerzen im rechten Oberarm erfolgt. Hier kam es aber offensichtlich zu einem Missverständnis, und es wurde davon ausgegangen, dass die Schmerzen den zuvor frakturierten Arm betreffen würden, sodass lediglich dieser Arm geröntgt wurde. Hierbei zeigte sich eine adäquate Kallusbildung.

Aufgrund der erneuten Fraktur eines großen Röhrenknochens innerhalb weniger Monate und der nichtplausiblen Anamnese wurde der Verdacht auf eine Kindeswohlgefährdung geäußert. Zusammen mit der Kinderschutzgruppe wurde das weitere Vorgehen festgelegt. In der Röntgenuntersuchung nach Leitlinie (Schädel, obere und untere Extremität, Wirbelsäule, Thorax- und Beckenübersicht, [3]) wurden zusätzlich zur Oberschenkelfraktur Frakturen beider Oberarme unterschiedlichen Alters sowie mehrere Rippenfrakturen festgestellt (• Abb. 1a-d). Eine Schädel-MRT 

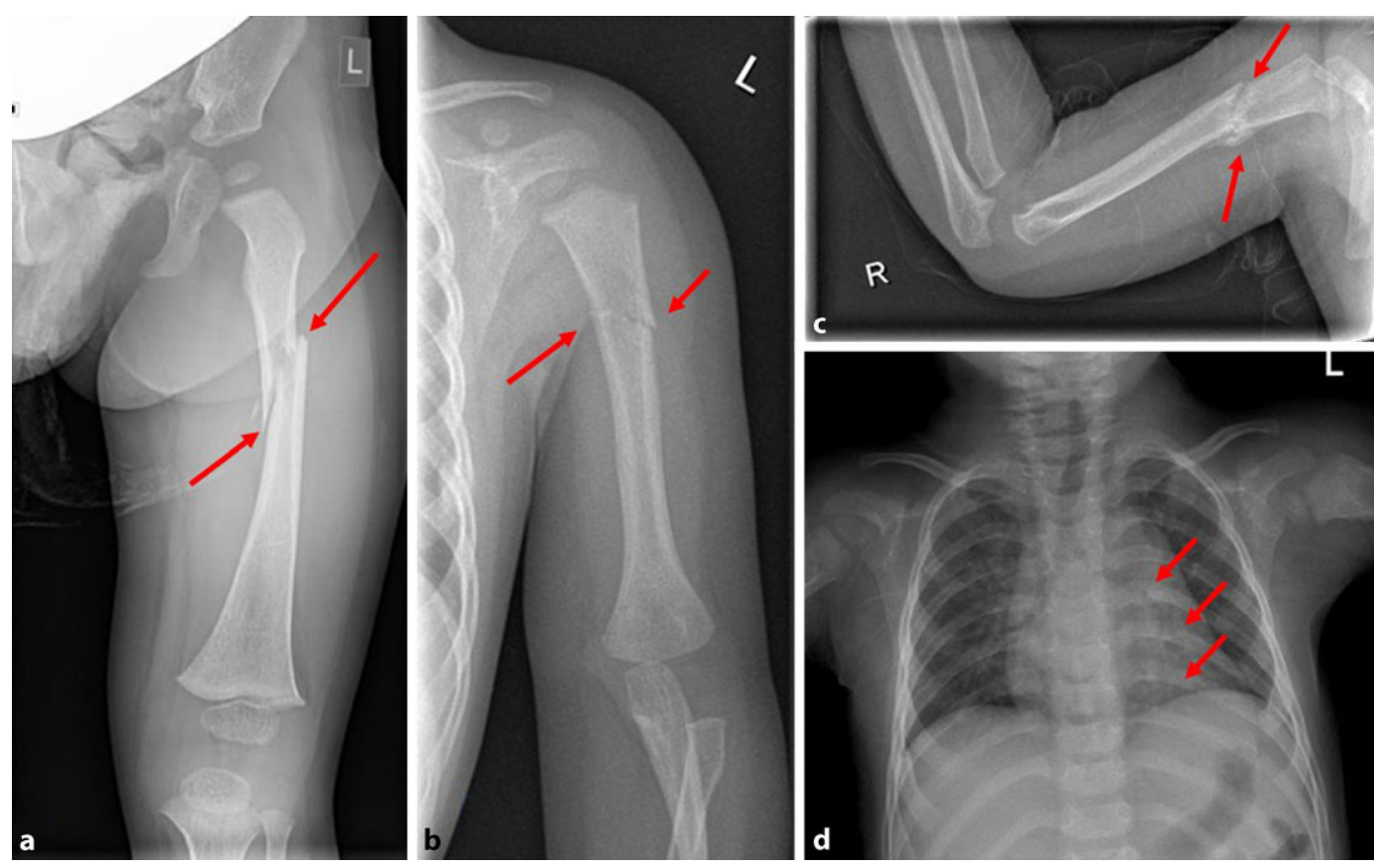

Abb. $1 \triangleleft$ Röntgendiagnostik, Auszug aus dem Röntgenskelettscreening; rote Pfeile markieren jeweils den Frakturspalt:a linkerOberschenkel mit proximaler Femurschaftfraktur, b linker Oberarm 2 Mon. zuvor mit proximaler Humerusschaftfraktur, c rechter Oberarm mit anamnestisch zum Zeitpunkt des Röntgenbildes ca. 3 Wochen alter Fraktur, d Thorax: Rippenserienfraktur der 6 . bis 8. Rippe links dorsal, Alter wenige Wochen. Fraglich auch rechts dorsal im Rippenbereich Frakturen

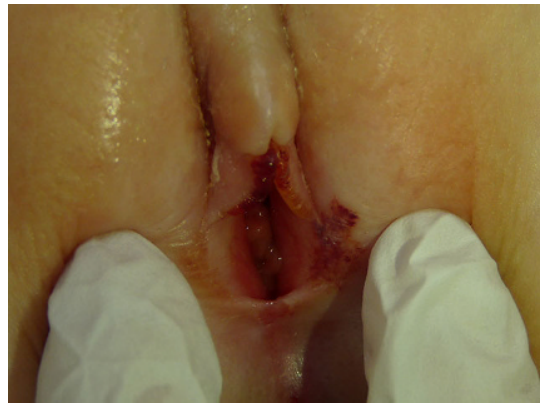

Abb. 2 \ Unterblutung der kleinen Labie rechts sowie eine punktförmige Unterblutung am Introitus zwischen $3 \mathrm{Uhr}$ und $5 \mathrm{Uhr}$

ebenso wie eine augenärztliche Untersuchung zum Ausschluss retinaler Blutungen waren unauffällig. Zusätzlich wurde ein genetisches Panel zur Diagnostik einer Osteogenesis imperfecta durchgeführt. Mit dem Ergebnis konnte jedoch erst eine bis 2 Wochen später gerechnet werden.

Es erfolgte die Kontaktaufnahme zum zuständigen Jugendamt und zur Rechtsmedizin München, und noch am gleichen Tag fand das erste von mehreren Helfergesprächen mit einem Dolmetscher statt. Den Eltern wurde der Verdacht der Kindesmisshandlung eingehend erläutert. Aufgrund des Gesamtbildes wurde vom Jugendamt die Indikation zur Inobhutnahme bis zur endgültigen Klärung des Sachverhaltes gestellt und das Kind 4 Tage nach Klinikaufnahme in eine Pflegefamilie entlassen. Die Geschwister blieben zunächst in der Familie; bei innen konnten keine eindeutig misshandlungsverdächtigen äuBeren Verletzungen festgestellt werden.

Nach weiteren 20 Tagen traf der humangenetische Befund ein; beim Patienten konnte eine Mutation im SERPINF1Gen, passend zu einer Osteogenesis imperfecta Typ 6, nachgewiesen werden. Dieser Befund wurde am gleichen Tag dem Jugendamt, der Pflegefamilie und den Eltern mitgeteilt, und das Kind konnte umgehend zurück in seine Familie verbracht werden. Im Verlauf wurde eine Konsanguinität der Eltern bekannt. Eine humangenetische Untersuchung zeigte bei beiden Eltern einen heterozygoten Nachweis der oben genannten Mutation.

\section{Fall 2}

Nach einer Vorstellung beim Kinderarzt wurde ein 8-jähriges Mädchen zunächst zum niedergelassenen Gynäkologen und dann in die Ambulanz der Kinderklinik verwiesen. Grund für die Vorstellung war der V.a. eine genitale Verletzung. Seit etwa einer Woche hatte die Patientin Schmerzen im Genitalbereich angegeben, und bereits zuvor war der Mutter ein Harnträufeln aufgefallen. Am Vorabend der Vorstellung waren der Mutter eine Verletzung und eine Schwellung der Schamlippen aufgefallen. Ein Trauma war der Mutter zunächst nicht erinnerlich; auf Nachfragen wurde ein mögliches Trauma auf einer Wippe vor einigen Tagen angegeben. Es erfolgte die ausführliche Untersuchung durch eine Frauenärztin der Klinik im Beisein einer Kinderärztin aus dem Team der Kinderschutzgruppe. Es zeigte sich ein ausgeprägter Befund: Zu sehen waren eine Unterblutung der kleinen Labie rechts sowie eine punktförmige Unterblutung am Introitus zwischen $3 \mathrm{Uhr}$ und $5 \mathrm{Uhr}$. Im Analbereich stellte sich bei $12 \mathrm{Uhr}$ eine Fissur dar ( A Abb. 2). Das Hymen zeigte keine frischen Einrisse. Es wurden DNAAbstriche vom Introitus entnommen. Im Gespräch mit der Psychologin zeigte sich eine harmonisch erscheinende Interaktion der Mutter mit dem Mädchen, und das Mädchen zeigte ein unauffälliges altersentsprechendes Verhalten. Die Mutter drängte auf Entlassung, es wurde bei unklarer Diagnose jedoch vor Entlassung das Jugendamt einvernehmlich involviert.

Aufgrund des Missbrauchsverdachtes erfolgte ein anonymisiertes Konsil durch die Rechtsmedizin München, inklusive Fotodokumentation. Hier bestätigte sich der V.a. einen ausgeprägten Lichen sclerosus et atrophicans. Durch die poröse Oberhaut konnte die Haut dabei bereits bei einem minimalen Trauma mit Blutungen einreiBen. Ein sexueller Missbrauch konnte perse nicht ausgeschlossen werden, erschien in der Gesamtzusammenschau aber unwahr- 
scheinlich. Es zeigten sich keine fremden DNA-Spuren.

\section{Fall 3}

Ein 4 Monate altes Kind wurde von den Eltern aufgrund eines unklaren Hämatoms am linken Handrücken vorgestellt. Eine Ursache für das Hämatom in Form eines Traumas war nicht erinnerlich. Im Rahmen der ansonsten unauffälligen Untersuchung fiel zudem auf, dass das Kind beim Hochheben unter den Achseln links empfindlich war. Im Röntgen wurde der Verdacht auf eine Fraktur gestellt. Bei dem schon länger zurückliegenden Fall wurde zu diesem Zeitpunkt zur Suche von älteren Frakturen anstelle eines Röntgenskelettscreenings noch eine Skelettszintigraphie durchgeführt. Hier zeigten sich keine Auffälligkeiten. Im Rahmen der möglichen Differenzialdiagnosen wurde auch der Verdacht auf eine Kindesmisshandlung mit den Eltern diskutiert.

Im Rahmen einer ausführlicheren Labordiagnostik zeigte sich die PTT mit $89 \mathrm{~s}$ deutlich verlängert, der Quick-Wert war $68 \%$, und der INR-Wert lag bei 1,3. Im weiteren Verlauf konnte eine schwere Hämophilie B mit Restaktivität von Faktor IX $<1 \%$ nachgewiesen werden. Die Familienanamnese war bezüglich hämatologischer Erkrankungen unauffällig.

Im weiteren Verlauf wurde das Kind immer wieder zur akuten Substituierung von Blutgerinnungsfaktor IX nach Trauma (Sturz aus Babyschale, Sturz vom Wickeltisch) vorgestellt.

\section{Diskussion}

\section{Abgestuftes Vorgehen bei Kinderschutzfällen und mögliche "pitfalls"}

Werden den Ärzten und Ärztinnen in der Kinderklinik gewichtige Anhaltspunkte für die Gefährdung des Wohls eines Kindes bekannt, schreibt $\S 4$ des Gesetzes zur Kooperation und Information im Kinderschutz (KKG) ein abgestuftes Verfahren vor, an dessen Ende unter bestimmten Voraussetzungen eine Mitteilung an das Jugendamt steht. Im Vorfeld soll die Situation mit dem Kind und den Personensorgeberechtigten erörtert und auf die
Inanspruchnahme von Hilfen hingewirkt werden ( $§ 4$ Abs. 1 KKG). Zur Einschätzung einer möglicherweise bestehenden Kindeswohlgefährdung haben Kinderärzte und Kinderärztinnen die Möglichkeit, sich durch eine „insoweit erfahrene Fachkraft" beraten zu lassen, sofern die übermittelten Daten pseudonymisiert werden ( $\$ 4$ Abs. 2 KKG). In diesem Zusammenhang können sie sich auch an eine rechtsmedizinische Stelle [1] wenden.

Irrtümer in beide Richtungen, nämlich entweder die Misshandlung oder den Missbrauch eines Kindes oder Jugendlichen zu übersehen oder aber diese fälschlicherweise zu diagnostizieren und hierdurch eine Intervention durch das Jugendamt zu initiieren, können Folgen für die gesamte Familie haben. Im Besonderen ist die Inobhutnahme durch das Jugendamt eine Intervention, die mit einer abrupten Trennung des Kindes von seinen engsten Bezugs- bzw. Bindungspersonen und somit, v.a. bei Kleinkindern, mit psychischen Belastungen einhergeht [4]. Speziell bei jüngeren Kindern dauerten Inobhutnahmen im Mittel um die 30 Tage [10]. Angesichts der plötzlichen Trennung des Kindes von seinen Bezugspersonen und der Tatsache, dass ihm in dieser Situation keine andere, vertraute, Bezugsperson zur Verfügung steht, sind die mit dieser Situation verbundenen physiologischen (erhöhte Cortisolausschüttung) und emotionalen Belastungen (depressive Reaktionen) für kleine Kinder signifikant [10]. Ist die Inobhutnahme also einerseits eine wichtige Maßnahme, um Kinder und Jugendliche vor weiteren Gefährdungen zu schützen, sollten andererseits ungerechtfertigte Inobhutnahmen unbedingt vermieden werden. Dieser Sachverhalt weist auf die Diffizilität der Kinderschutzarbeit und die auch für Ärzte und Ärztinnen in diesem Tätigkeitsfeld bestehenden Herausforderungen hin.

\section{Falsch-positive Fälle und mögliche Differenzialdiagnosen}

In einer Untersuchung des Projektes Kinderschutz der Rechtsmedizin Hannover wurden im Zeitraum von 2010 bis 2013 $30,3 \%$ der Verdachtsfälle seitens der erstbehandelnden Ärzte entkräftet, weil entweder ein Trauma, eine Normvarian- te oder eine medizinische Erkrankung in Betracht kam [9]. In einem rezenten publizierten Fall wurde in Ontario, Kanada, ein lebenslängliches Urteil gegen den Onkel eines 4-jährigen Kindes aufgehoben. Das Kind wurde leblos im Bett aufgefunden, der Onkel fungierte zu dieser Zeit als „Babysitter". Postmortale Artefakte, wie eine postmortal aufgetretene Blutung im Halsbereich sowie eine postmortal aufgetretene anale Dilatation, wurden im ursprünglichen Prozess als anale Vergewaltigung mit Würgen und Tod durch Asphyxie gewertet [7]. Die eigentliche Todesursache des Kindes konnte nicht festgestellt werden, aber natürliche Ursachen wie Long-QT-Syndrom, Epilepsie oder Anaphylaxie wurden in Betracht gezogen. Der Onkel war bis zur Aufhebung des Urteils 12 Jahre inhaftiert.

Differenzialdiagnosen bei Verdacht auf Kindesmisshandlung sind mitunter sehr seltene Erkrankungen. So können z.B. Erweiterungen des Subarachnoidalraums und subdurale Blutungen eine Glutaracidurie Typ I (u.U. mit Retinablutungen), eine Störung des Kupfertransportes (Menkes-Syndrom) oder einen Cobalamin-CMangel als Ursache haben [8]. Als Ursache für Frakturen kommen eine Osteogenesis imperfecta oder ein alimentär bedingter Vitamin-D-Mangel in Betracht [6]. Longitudinale Streifen am Rücken, welche u.U. auch vernarben, können eine harmlose Bindegewebsveränderung in der Pubertät als Ursache haben (Striae distensae). Mongolenflecke, welche üblicherweise im Kleinkindalter abblassen, können mit Hämatomen verwechselt werden [8]. Genitale Verletzungen (Einrisse) und Blutungen können durch hypotrophe Hautveränderungen im Rahmen eines Lichen sclerosus wie im beschriebenen Fall bedingt sein. Hämatome wiederum können durch eine Gerinnungsstörung hervorgerufen werden, welche nicht in der Routinediagnostik erkennbar ist, z. B. ein WillebrandSyndrom oder seltenere Thrombozytenfunktionsstörungen (M. Glanzmann) [2]. Siehe hierzu auch die entsprechende S3Leitlinie Kinderschutz [3]. 
Kindeswohlgefährdung und Situation der Eltern im Zusammenhang mit medizinischen Fehleinschätzungen

In den hier beschriebenen Fällen konnte eine Kindesmisshandlung letztendlich ausgeschlossen werden, dennoch sprach in den beschriebenen Fällen die initiale Konstellation für eine Kindeswohlgefährdung. Der klinische Verlauf mit initialem Verdachtsmoment sowie dem anschließenden Einbezug des Jugendamtes und des Familiengerichtes führten jedoch zu Belastungen für Eltern und Kinder [5].

Die Mutter des Kindes mit Osteogenesis imperfecta, selbst durch Flucht traumatisiert und ohne Deutschkenntnisse, war plötzlich damit konfrontiert, dass ihr Kind durch das Jugendamt in Obhut genommen und in einer Pflegefamilie untergebracht wurde. Das Kind war insgesamt 21/2 Wochen nicht bei der Familie. Anfängliche Überlegungen, auch die anderen Kinder zum Schutz aus der Familie zu nehmen, wurden nicht umgesetzt. Im Anschluss beschrieben die Eltern dies als eine extrem traumatisch empfundene Situation. Die Familie wurde für die Therapie an die Klinik angebunden.

Die Diagnosestellung Lichen sclerosus erfolgt in der Regel anhand des klinischen Erscheinungsbildes mit pergamentpapierartigen Veränderungen der Haut im Anogenitalbereich. Im beschriebenen Fall war aufgrund der akuten vaginalen Verletzungen eine eindeutige Zuordnung zunächst erschwert. Auf dringenden Wunsch der Mutter wurde das Kind dennoch vor Klärung der endgültigen Diagnose nach Hause entlassen, jedoch wurde vor der Entlassung das Jugendamt mit dem Einverständnis der Mutter involviert. Dieses abgestufte Vorgehen erscheint auch im Nachhinein gerechtfertigt. Man wollte von Klinikseite mit der Involvierung des Jugendamtes sicherstellen, dass das Kind vor erneutem sexuellen Missbrauch geschützt wird. Nach der Diagnosestellung Lichen sclerosus, dem Erhalt aller Befunde und der Rückmeldung der Rechtsmedizin erfolgte ein erneutes Gespräch mit der Mutter, in dem die Diagnose sowie mögliche Therapien erläutert und eine kindergynäkologische Anbindung empfohlen wurden. Die Mutter zeigte sich in dem Gespräch emotional sehr belastet. Der Befund des Kindes heilte unter topischer Steroidtherapie ab.

\section{Fazit für die Praxis}

Die vorgestellten Fälle hatten als Ursache für vermeintliche nichtakzidentelle Verletzungsmuster eine genetisch gesicherte Osteogenesis imperfecta Typ 6, einen Lichen sclerosus und eine Hämophilie B. Die Dunkelziffer der nichtdiagnostizierten Fälle von Kindesmisshandlung ist vermutlich um ein vielfaches höher als die Fälle, welche falsch-positiv diagnostiziert wurden. Dennoch müssen medizinische Diagnosen als Differenzialdiagnose unbedingt ausgeschlossen werden. Hierfür ist die Zusammenarbeit der jeweiligen Fachdisziplinen unabdingbar. Falls die klinische oder genetische Diagnostik länger dauert als der stationäre Aufenthalt, sollte das zuständige Jugendamt in Rücksprache mit der Kinderschutzgruppe der Klinik festlegen, mit welchen Maßnahmen der Schutz des Kindes bis zum Abschluss der Diagnostik gewährleistet werden kann. In der Regel kann ein Kind nicht über Wochen bis zum Erhalt der genetischen Diagnostik stationär in der Klinik bleiben, u.U. wäre aber auch ein abgestuftes Vorgehen des Jugendamtes denkbar, um eine Inobhutnahme zu vermeiden.

Um die eingangs beschriebenen Irrtümer in beide Richtungen - das Kind verbleibt im misshandelnden Umfeld oder wird ungerechtfertigt in Obhut genommen - bestmöglich zu vermeiden, sollte die Diskussion eines Falles in interdisziplinär und interprofessionell besetzten Kinderschutzgruppen obligatorisch sein und die Gründung von Kinderschutzgruppen an Kinderkliniken weiter vorangetrieben werden. Notwendige Differenzialdiagnosen können auf diesem Weg ins Gespräch gebracht werden; auch erleichtert eine Kinderschutzgruppe die oftmals notwendige Kooperation mit dem Jugendamt und der Polizei. Die Konsultation der Rechtsmedizin ist in vielen Fällen hilfreich, um die Diagnose entsprechend einzugrenzen oder aber um alternative medizinische Diagnosen anzubieten.

\section{Korrespondenzadresse}

PD Dr. med. habil. Gerhard K. Wolf

Kinderklinik Traunstein, Kliniken Südostbayern, akademisches Lehrkrankenhaus der LMU München

Cuno-Niggl-Str. 3, 83278 Traunstein,

Deutschland

gerhard.wolf@Imu.de

Funding. Open Access funding enabled and organized by Projekt DEAL.

\section{Einhaltung ethischer Richtlinien}

Interessenkonflikt. S. Röding, A. Dittmann-Wolf, D. Lackner, C. Blume, A. Hasse, E. Mützel und G.K. Wolf geben an, dass kein Interessenkonflikt besteht.

Für diesen Beitrag wurden von den Autoren keine Studien an Menschen oder Tieren durchgeführt. Für die aufgeführten Studien gelten die jeweils dort angegebenen ethischen Richtlinien. Auf eine Einwilligung der Patienten zur Bilddokumentation wurden im LMUEthikvotum unter Anonymisierung der Patienten verzichtet.

Open Access. Dieser Artikel wird unter der Creative Commons Namensnennung 4.0 International Lizenz veröffentlicht, welche die Nutzung, Vervielfältigung Bearbeitung, Verbreitung und Wiedergabe in jeglichem Medium und Format erlaubt, sofern Sie den/die ursprünglichen Autor(en) und die Quelle ordnungsgemäß nennen, einen Link zur Creative Commons Lizenz beifügen und angeben, ob Änderungen vorgenommen wurden.

Die in diesem Artikel enthaltenen Bilder und sonstiges Drittmaterial unterliegen ebenfalls der genannten Creative Commons Lizenz, sofern sich aus der Abbildungslegende nichts anderes ergibt. Sofern das betreffende Material nicht unter der genannten Creative Commons Lizenz steht und die betreffende Handlung nicht nach gesetzlichen Vorschriften erlaubt ist, ist für die oben aufgeführten Weiterverwendungen des Materials die Einwilligung des jeweiligen Rechteinhabers einzuholen.

Weitere Details zur Lizenz entnehmen Sie bitte der Lizenzinformation auf http://creativecommons.org/ licenses/by/4.0/deed.de.

\section{Literatur}

1. Bayerisches Staatsministerium für Familie Arbeit und Soziales Kinderschutzambulanz Eine bayernweite Anlaufstelle zur Beratung bei Verdacht auf Kindesmisshandlung. https://www.stmas. bayern.de/kinderschutz/kinderschutzambulanz. Zugegriffen: 1.4.2021

2. Bidlingmaier C, Olivieri M, Kurnik K (2012) Hautblutungen bei Kindern. Monatsschr Kinderheilkd 6:538-544

3. Blesken M, Franke I, Freiberg J et al (2019) Langfassung der Leitlinie „Kindesmisshandlung, -missbrauch, -vernachlässigung unter Einbindung der Jugendhilfe und Pädagogik (Kinderschutzleitlinie)". https://www.awmf.org/leitlinien/detail//l/ 027-069.html. Zugegriffen: 2.4.2021

4. Bowlby J (2006) Verlust, Trauer und Depression. Reinhardt,

5. Dettenborn H (2003) Die Beurteilung der Kindeswohlgefährdung als Risikoentscheidung. FPR 06:293-299

6. Pickhardt C, Urban R, Körber F et al (2016) Seltene Differentialdiagnosen bei Verdacht auf Kindesmisshandlung. Monatsschr Kinderheilkd 11:1020-1024

7. Pollanen MS (2012) Forensic pathology and the miscarriage of justice. Forensic Sci Med Pathol 8:285-289

8. Scholl-Bürgi $S$, Kapelari $K$, Michel $M$ et al (2016) Angeborene Stoffwechselstörungen in der Differenzialdiagnose von Kindesmisshandlung. Pädiatr Prax 86:273-283 
9. Todt M, Maciuga A, Derbertin AS (2014) „Projekt Kinderschutz" in Niedersachsen. Rechtsmedizin 24:399-404

10. Ziegenhain U, Fegert J, Petermann F et al (2014) Inobhutnahme und Bindung. Kindh Entwickl 23:248-259

\section{A retrospective analysis of differential diagnoses in cases of suspected child abuse. Child abuse, no doubt—or not?}

Background: The diagnosis of non-accidental trauma or child abuse can lead to errors in both directions: either child abuse can go undetected or child abuse can be wrongfully diagnosed, if other differential diagnoses were not correctly taken into account. Such diagnostic errors can lead to continued child abuse in the home setting, or that the child is removed from the family without reason and the parents are falsely accused of child abuse.

Methods: Cases of child abuse from the last 10 years were retrospectively reviewed in order to identify false positive cases. The study and publication were reviewed and approved by the ethics committee of the Ludwig-Maximilians-University Munich. Results: The cases where child abuse was wrongfully suspected had final diagnoses such as osteogenesis imperfecta type 6, lichen sclerosus, and hemophilia B. In all cases, local child protective services were involved, and in some cases the family court. In one case, the child was temporarily removed from the family.

Discussion: The child protection team can review necessary differential diagnoses and coordinate the collaboration with the local child protective services, the police, and the family courts. Removing a child from the family can be an effective measure to protect the child from further abuse in the case that abuse actually happened but unjustified placement in a foster family, even temporarily, must be avoided by all means, as this measure may unnecessarily traumatize children and parents. Consultation with forensic medicine may be helpful to establish the correct diagnosis.

\section{Keywords}

Child abuse $\cdot$ Child protection team · Osteogenesis imperfecta $\cdot$ Lichen sclerosus $\cdot$ Hemophilia 